\title{
Closed-Form Optimal Strategies of Continuous-Time Options with Stochastic Differential Equations
}

\author{
Wei Yan \\ Research Institute of Petroleum Exploration and Development, PetroChina, Beijing 100083, China \\ Correspondence should be addressed to Wei Yan; yanwei123456@petrochina.com.cn
}

Received 18 October 2016; Revised 15 March 2017; Accepted 24 April 2017; Published 2 July 2017

Academic Editor: Pietro De Lellis

Copyright (C) 2017 Wei Yan. This is an open access article distributed under the Creative Commons Attribution License, which permits unrestricted use, distribution, and reproduction in any medium, provided the original work is properly cited.

\begin{abstract}
A continuous-time portfolio selection with options based on risk aversion utility function in financial market is studied. The different price between sale and purchase of options is introduced in this paper. The optimal investment-consumption problem is formulated as a continuous-time mathematical model with stochastic differential equations. The prices processes follow jumpdiffusion processes (Weiner process and Poisson process). Then the corresponding Hamilton-Jacobi-Bellman (HJB) equation of the problem is represented and its solution is obtained in different conditions. The above results are applied to a special case under a Hyperbolic Absolute Risk Aversion (HARA) utility function. The optimal investment-consumption strategies about HARA utility function are also derived. Finally, an example and some discussions illustrating these results are also presented.
\end{abstract}

\section{Introduction}

Pricing theory of options is an important research field. In 1973, Black and Scholes published an innovative paper [1]. In this paper, a theoretical valuation formula is derived. The well-known Black-Scholes formula is deduced. This formula's creative point does not lie in the investor's preferences but a risk-neutral method with a risk-free asset. It is applied in many fields extensively. However, the varieties of options become more and more. The theory of options pricing must be improved. Up to now, the most options pricing models are extended based on the Black-Scholes formula. Merton [2], Jeanblanc-Picque and Pontier [3], and Lin and Ye [4] added the jump process to the option pricing formula; Barron and Jensen [5] spread the formula while the interest rate has difference between the deposition and loan. In addition, there are a lot of other pricing models, such as Cox et al. [6].

Nowadays, the objective is to earn wealth with portfolio selection although the options pricing is still a central research topic. We could regard the options as a risk asset, like stocks. As a risk asset, options catch more and more attention in financial market. There exists options trading in many exchanges all over the world, such as $\mathrm{CBOE}$ (Chicago Board Option Exchange), PHLX (Philadelphia
Stock Exchange), AMEX (American Stock Exchange), PSE (Pacific Stock Exchange), NYSE (New York Stock Exchange). In most options exchange, they follow the market maker rules. The price of purchasing or selling an options contract is equivalent to the price at which the market maker wants to sell or purchase it. Therefore, the options have the same position with other underlying assets. However, for the general investment-consumption problem analysis approach, there has been few literatures involving options trading. In this paper, the options contract can be added to the optimal investment-consumption problem.

One of the frequent questions in finance is how to allocate a certain amount of money in different assets and at what time instant. The earliest approach to consider the optimal portfolio problem is so called mean-variance criterion. It was pioneered by Markowitz [7] and has been playing a critical role in the theory of portfolio selection. It has also gained widespread acceptance as a practical tool for portfolio optimization. However, it is a single-period model which makes one-off decision at the beginning of the period and holds on until the end of the period. Gradually, researchers extended the above single-period model to continuous-time models $[8,9]$. An optimal consumption-investment problem has been formulated. By applying results from stochastic control 
theory to the optimal portfolio problem, explicit solutions have been obtained for some special cases. Many researches about continuous-time portfolio selection appeared [10-15].

In general literatures, continuous-time investment-consumption model is modeled by assuming a market in which the evolution of assets prices is described by a continuous process. However, as pointed by Merton $[2,16]$ and Jarrow and Rudd [17], the actual price of stock is not continuous because there is some external information affecting it. In fact, in addition to the continuous process based on Brown motion, the analysis of price evolution does reveal sudden and rare breaks logically accounted for by exogenous events on information. Such a behavior from probabilistic point of view is naturally modeled by a point process. This process governed by Brownian motion and point process is called jump-diffusion process. It is a discontinuous price process. Some concrete results $[2,18]$ prove that there are some jump processes in reality. A stochastic exchange rate with jumpdiffusion processes is concerned in the mean-variance portfolio selection [19]. However, it is a linear-quadratic model. The calculation process and results may not be complicated. We hope that the objective function has a general form like a Hyperbolic Absolute Risk Aversion (HARA) utility function of this paper. Moreover, there is a nondifferentiable point in the capital process in the following paper. These lead to the calculation process and results that are more complicated. We want to obtain some more general results in a sense.

In financial markets, the price of options has difference between selling and purchasing. Different from the classical Merton model, we make a hypothesis on the European call option trading price that the purchasing price is usually bigger than selling price. This simple hypothesis is only a constraint between purchasing and selling. The investor can add some other constraints. However, more complicated constraints would prevent the derivation of closed-form analytical solutions. Therefore, we add this simple constraint to the unconstrained classical Merton model.

This paper is concerned with the optimal investmentconsumption portfolio selection of options with different price between sale and purchase based on a risk aversion utility function in financial markets. We study how the different prices affect the strategies of investment-consumption. So the problem is formulated as a continuous-time mathematical model. Considering the difference price of the options, the paper is more complex in the price process than traditional Merton model. Besides, these pricing processes follow jumpdiffusion processes (Weiner process and Poisson process). The model contains discontinuous price processes. Then, the corresponding Hamilton-Jacobi-Bellman (HJB) equation of the problem is represented and its solutions are obtained in different conditions. Finally, the above results are applied to a special case in which the objective function is a Hyperbolic Absolute Risk Aversion (HARA) utility function. Under the above objective function, we can obtain a closed-form solution. We can avoid the computational complexity in numerical calculation, getting the investment strategy more effectively and concisely.

\section{Mathematical Model}

In this section, we formulate a mathematical model. There are 3 assets being traded on a finite horizon $[0, T]$. They are one bond, one risky asset,and its corresponding options. In this paper, we only consider European call option contracts like other kinds of similar risky assets.

One asset is a bond, whose price $S_{0}(t)$ evolves according to the differential equation

$$
\begin{aligned}
\mathrm{d} S_{0}(t) & =S_{0}(t) r(t) \mathrm{d} t, \quad t \in[0, T], \\
S_{0}(0) & =s_{0},
\end{aligned}
$$

where $r(t)$ is the interest rate of the bond.

Similar to Merton [16], the price process $F(t)$ of the risk asset should satisfy the following stochastic differential equation governed by a Weiner process and a Poisson process:

$$
\begin{aligned}
\mathrm{d} F(t) & =F\left(t_{-}\right)[\omega(t) \mathrm{d} t+\theta(t) \mathrm{d} W(t)+\zeta(t) \mathrm{d} N(t)], \\
t \in[0, T], & \\
F(0) & =f_{0}>0,
\end{aligned}
$$

where $\Phi(t)$ is the appreciation rate and $\theta(t)$ and $\zeta(t)$ are the volatility coefficient; $t_{-}$is $s<t$ while $s \rightarrow t$. We consider a financial market which is subject to uncertainty that enters through a one-dimensional Weiner process $W(t)$ on its canonical space $\left(\Omega^{W}, L^{W}, P^{W}\right)$ and a one-dimensional Poisson process $N(t)$ on its canonical space $\left(\Omega^{N}, L^{N}, P^{N}\right)$ with intensity equal to $\lambda(t)$. By Jeanblanc-Picque and Pontier [3], the compensated Poisson process defined by $M(t)=$ $N(t)-\int_{0}^{T} \lambda(s) \mathrm{d} s(t \in[0, T])$ is a martingale. We denote the $P^{W}$-augmentation of $\sigma(W(s): 0 \leq s \leq t)$ by $\left\{L_{t}{ }^{W}\right\}$ and the $P^{N}$-augmentation of $\sigma(N(s): 0 \leq s \leq t)$ by $\left\{L_{t}{ }^{N}\right\}$. Let $(\Omega, L, P)=\left(\Omega^{W} \otimes \Omega^{N}, L^{W} \otimes L^{N}, P^{W} \otimes P^{N}\right)$ denote the product space. On this space, $W(t)$ and $N(t)$ are independent. Moreover, the coefficients $r(t), \varrho(t), \theta(t), \zeta(t)$, and $\lambda(t)$ in the stochastic differential equations are deterministic, Borelmeasurable, and bounded.

Applying Ito's formula [20] to the purchasing price $Y(t, F)$ of the corresponding European call options, we obtain

$$
\begin{aligned}
& \mathrm{d} Y(t, F) \\
& =\left(Y_{t}+F(t) \omega(t) Y_{F}+\frac{1}{2} F(t)^{2} \theta(t)^{2} Y_{F F}\right) \mathrm{d} t \\
& \quad+F(t) \theta(t) Y_{F} \mathrm{~d} W(t) \\
& \quad+[Y(t, F(t)+F(t) \zeta(t))-Y(t, F(t))] \mathrm{d} N(t), \\
& t \in[0, T], \\
& Y(T, F(T))=(F(T)-q)^{+},
\end{aligned}
$$

where $Y_{t}=\partial Y(t, F) / \partial t, Y_{F}=\partial Y(t, F) / \partial F, Y_{F F}=\partial^{2} Y(t$, $F) / \partial F^{2},(F(T)-q)^{+}=\{F(T)-q$, if $F(T) \geq q$; 0 , if $F(T)<q\}$, and $q$ is strike price (the rest of the partial deferential symbols of this paper are the same as $\left.Y_{t}\right) . Y(t, F)$ is actually a function 
of futures prices $F(t)$ and time $t$. $F(t)$ should be a unitary part in $Y(t, F)$. When we apply Ito's formula, we should apply it to the corresponding process $Y(t, F(t))$, which is defined in terms of the function $Y$ and the process $F(t)$.

We assume that the price of options is different when selling or purchasing. The purchasing price is usually bigger than selling price. We set the selling price as $G(t, F)=$ $\alpha(t) Y(t, F)$, where $0<\alpha(t)<1$. Moreover, for simplicity it is assumed that $\alpha(t)$ is a monotone nondecreasing function differentiable with respect to time $t$. Then $G(t, F)$ satisfies the following equation:

$$
\begin{aligned}
& \mathrm{d} G(t, F) \\
& =\left(G_{t}+F(t) \omega(t) G_{F}+\frac{1}{2} F(t)^{2} \theta(t)^{2} G_{F F}\right) \mathrm{d} t \\
& +F(t) \theta(t) G_{F} \mathrm{~d} W(t) \\
& +[G(t, F(t)+F(t) \zeta(t))-G(t, F(t))] \mathrm{d} N(t), \\
& t \in[0, T], \\
& G(T, F(T))=\alpha(T)(F(T)-q)^{+} .
\end{aligned}
$$

Substituting $G(t, F)=\alpha(t) Y(t, F)$ into the above equation, we obtain

$$
\begin{aligned}
& \mathrm{d} G(t, F)=\left(\dot{\alpha}(t) Y(t, F)+\alpha(t) Y_{t}\right. \\
& \left.+\alpha(t) F(t) \omega(t) Y_{F}+\frac{1}{2} \alpha(t) F(t)^{2} \theta(t)^{2} Y_{F F}\right) \mathrm{d} t \\
& +\alpha(t) F(t) \theta(t) Y_{F} \mathrm{~d} W(t)+\alpha(t) \\
& \cdot[Y(t, F(t)+F(t) \zeta(t))-Y(t, F(t))] \mathrm{d} N(t), \\
& \quad t \in[0, T], \\
& G(T, F(T))=\alpha(T)(F(T)-q)^{+} .
\end{aligned}
$$

Let $\pi_{0}(t), \pi_{1}(t)$, and $\pi_{2}(t)$ be the amount allocated to the bond, risky asset, and options, respectively, which may be positive or negative. It is assumed that $\omega_{0}(t), \omega_{1}(t)$, and $\omega_{2}(t)$ are the shares of the above derivatives mentioned, respectively, and then $\pi_{0}(t)=\omega_{0}(t) S_{0}(t), \pi_{1}(t)=\omega_{1}(t) F(t)$, $\pi_{2}(t)=\omega_{2}(t) Y(t, F)$, or $\pi_{2}(t)=\omega_{2}(t) G(t, F)$.

It is assumed that an investor has initial capital $x(t)=$ $x_{0}(t)(t=0)$.

When an investor is purchasing option contracts $\left(\pi_{2}(t)>\right.$ 0 ), we can get the differential equation as follows by considering the dynamic process of investment and consumption $\{c(t), 0 \leq t \leq T\}$ :

$$
\begin{aligned}
& \mathrm{d} x(t)=\omega_{0}(t) \mathrm{d} S_{0}(t)+\omega_{1}(t) \mathrm{d} F(t)+\omega_{2}(t) \mathrm{d} Y(t, F) \\
& -c(t) \mathrm{d} t=\left[x(t) r(t)+\pi_{1}(t)(\varrho(t)-r(t))\right. \\
& +\pi_{2}(t)
\end{aligned}
$$

$$
\begin{aligned}
& \cdot\left(\frac{Y_{t}+F(t) \omega(t) Y_{F}+(1 / 2) F^{2} \theta(t)^{2} Y_{F F}}{Y(t, F(t))}-r(t)\right) \\
& -c(t)] \mathrm{d} t+\left[\pi_{1}(t) \theta(t)+\pi_{2}(t)\right. \\
& \left.\cdot \frac{F(t) \theta(t) Y_{F}}{Y(t, F(t))}\right] \mathrm{d} W(t)+\left[\pi_{1}(t) \zeta(t)\right. \\
& +\pi_{2}(t) \\
& \left.\cdot \frac{Y(t, F(t)+F(t) \zeta(t))-Y(t, F(t))}{Y(t, F(t))}\right] \mathrm{d} N(t), \\
& x(0)=x_{0} .
\end{aligned}
$$

Set $b_{1}(t)=\omega(t), b_{2}(t)=\left(Y_{t}+F(t) \omega(t) Y_{F}+\right.$ $\left.(1 / 2) F^{2} \theta(t)^{2} Y_{F F}\right) /(Y(t, F(t))), \sigma_{1}(t)=\theta(t), \sigma_{2}(t)=$ $\left(F(t) \theta(t) Y_{F}\right) /(Y(t, F(t))), \varphi_{1}(t)=\zeta(t), \varphi_{2}(t)=(Y(t, F(t)+$ $F(t) \zeta(t))-Y(t, F(t))) /(Y(t, F(t)))$. Then, it can be obtained that

$$
\begin{aligned}
& \mathrm{d} x(t)=\left[x(t) r(t)+\pi_{1}(t)\left(b_{1}(t)-r(t)\right)\right. \\
& \left.\quad+\pi_{2}(t)\left(b_{2}(t)-r(t)\right)-c(t)\right] \mathrm{d} t+\left[\pi_{1}(t) \sigma_{1}(t)\right. \\
& \left.\quad+\pi_{2}(t) \sigma_{2}(t)\right] \mathrm{d} W(t)+\left[\pi_{1}(t) \varphi_{1}(t)\right. \\
& \left.\quad+\pi_{2}(t) \varphi_{2}(t)\right] \mathrm{d} N(t), \quad t \in[0, T], \\
& x(0)=x_{0} .
\end{aligned}
$$

When an investor is selling an option contract $\left(\pi_{2}(t) \leq 0\right)$, we can get the similar differential equation

$$
\begin{aligned}
& \mathrm{d} x(t)=\omega_{0}(t) \mathrm{d} S_{0}(t)+\omega_{1}(t) \mathrm{d} F(t)+\omega_{2}(t) \mathrm{d} G(t, F) \\
& -c(t) \mathrm{d} t=\left[x(t) r(t)+\pi_{1}(t)\left(b_{1}(t)-r(t)\right)\right. \\
& \left.+\pi_{2}(t)\left(b_{2}(t)-r(t)+\frac{\dot{\alpha}(t)}{\alpha(t)}\right)-c(t)\right] \mathrm{d} t \\
& +\left[\pi_{1}(t) \sigma_{1}(t)+\pi_{2}(t) \sigma_{2}\right] \mathrm{d} W(t) \\
& +\left[\pi_{1}(t) \varphi_{1}(t)+\pi_{2}(t) \varphi_{2}\right] \mathrm{d} N(t),
\end{aligned}
$$

$$
t \in[0, T]
$$

$$
x(0)=x_{0} .
$$

Remark 1. In this paper, we regard options as general risky assets just like stocks. Besides, it is assumed that $\alpha(t)$ is not constant, but a function of time $t$. 
Merging (7) and (8), it can be obtained that

$$
\begin{aligned}
& \mathrm{d} x(t)=\left[x(t) r(t)+\pi_{1}(t)\left(b_{1}(t)-r(t)\right)\right. \\
& +\pi_{2}(t)\left(b_{2}(t)-r(t)\right)-\left(\frac{\dot{\alpha}(t)}{\alpha(t)}\left(\pi_{2}(t)\right)^{-}\right) \\
& \quad-c(t)] \mathrm{d} t+\left[\pi_{1}(t) \sigma_{1}(t)+\pi_{2}(t) \sigma_{2}\right] \mathrm{d} W(t) \\
& \quad+\left[\pi_{1}(t) \varphi_{1}(t)+\pi_{2}(t) \varphi_{2}\right] \mathrm{d} N(t),
\end{aligned}
$$

$$
t \in[0, T]
$$

$$
x(0)=x_{0}
$$

where $\left(\pi_{2}(t)\right)^{-}=\left\{0\right.$, if $\pi_{2}(t)>0 ;-\pi_{2}(t)$, if $\left.\pi_{2}(t) \leq 0\right\}$.

Then, (9) can be transformed into the following equation:

$$
\begin{gathered}
\mathrm{d} x(t)=\left[x(t) r(t)+\pi(t)^{\prime}\left(b(t)-r(t) 1_{2}\right)\right. \\
\left.-\left[\frac{\dot{\alpha}(t)}{\alpha(t)}\left(\pi_{2}(t)\right)^{-}\right]-c(t)\right] \mathrm{d} t+\pi(t)^{\prime} \\
\cdot \sigma(t) \mathrm{d} W(t)+\pi(t)^{\prime} \varphi(t) \mathrm{d} N(t),
\end{gathered}
$$

$$
t \in[0, T],
$$

$$
x(0)=x_{0},
$$

where $1_{2}$ denotes a 2 -dimensional column vector with every entry being $1 ; b(t)=\left[b_{1}(t), b_{2}(t)\right]^{\prime}, \pi(t)=\left[\pi_{1}(t), \pi_{2}(t)\right]^{\prime}$, $\sigma(t)=\left[\sigma_{1}(t), \sigma_{2}(t)\right]^{\prime}, \varphi(t)=\left[\varphi_{1}(t), \varphi_{2}(t)\right]^{\prime} .\left(M^{\prime}\right.$ denotes the transpose of any matrix or vector $M$.)

The objective of an investor is to maximize the utility of consumption and terminal expected wealth.

$$
\begin{aligned}
& J\left(x, c, \pi_{1}, \pi_{2}\right) \\
& \quad=E\left[\int_{0}^{T} U_{1}(t, c(t)) \mathrm{d} t+U_{2}(T, x(T))\right],
\end{aligned}
$$

where the utility functions $U_{1}(\cdot)$ and $U_{2}(\cdot)$ are risk aversion functions.

\section{HJB Equation}

In this section, we will deduce the HJB function corresponding to (10) and (11) similar to Sage and White [21].

Firstly, we set the value function as follows:

$$
V(t, x)=\max _{c, \pi} E_{t, x}\left[\int_{t}^{T} U_{1}(s, c) \mathrm{d} s+U_{2}(T, x)\right] .
$$

Then, it can be obtained as follows by Bellman optimal principle that

$$
\begin{aligned}
& V(t, x)=\max _{c, \pi} E_{t, x}\left\{\int_{t}^{t+\Delta t} U_{1}(s, c) \mathrm{d} s\right. \\
& \left.+\int_{t+\Delta t}^{T} U_{1}(s, c) \mathrm{d} s+U_{2}(T, x)\right\}=\max _{c, \pi} E_{t, x} \\
& \quad \cdot\left\{\int_{t}^{t+\Delta t} U_{1}(s, c) \mathrm{d} s+V(t+\Delta t, x+\Delta x)\right\},
\end{aligned}
$$

where $E_{t, x}$ denotes the conditional expectation when the initial condition is given by $(t, x)$.

It can be obtained that

$$
\begin{aligned}
& V(t+\Delta t, x+\Delta x)=V(t, x)+V_{t} \Delta t+V_{x} \Delta x+\frac{1}{2} \\
& \cdot V_{t t}(\Delta t)^{2}+\frac{1}{2} V_{x x}(\Delta x)^{2}+V_{t x} \Delta t \Delta x, \\
& \Delta x(t)=\left[x(t) r(t)+\pi(t)^{\prime}\left(b(t)-r(t) 1_{2}\right)\right. \\
& \left.-\left[\frac{\dot{\alpha}(t)}{\alpha(t)}\left(\pi_{2}(t)\right)^{-}\right]-c(t)\right] \Delta t+\pi(t)^{\prime} \sigma(t) \Delta W(t) \\
& +\pi(t)^{\prime} \varphi(t) \Delta N(t)=[x(t) r(t) \\
& +\pi(t)^{\prime}\left(b(t)-r(t) 1_{2}+\lambda(t) \varphi(t)\right) \\
& \left.-\left[\frac{\dot{\alpha}(t)}{\alpha(t)}\left(\pi_{2}(t)\right)^{-}\right]-c(t)\right] \Delta t+\pi(t)^{\prime} \sigma(t) \Delta W(t) \\
& +\pi(t)^{\prime} \varphi(t) \Delta M(t) .
\end{aligned}
$$

Substituting the above two equations into (13), we can get

$$
\begin{aligned}
& V(t, x)=\max _{c, \pi} E_{t, x}\left\{U_{1}(t, c) \Delta t+V(t, x)+V_{t} \Delta t\right. \\
& \left.+V_{x} \Delta x+\frac{1}{2} V_{t t}(\Delta t)^{2}+\frac{1}{2} V_{x x}(\Delta x)^{2}+V_{t x} \Delta t \Delta x\right\} \\
& =\max _{c, \pi} E_{t, x}\left\{U_{1}(t, c) \Delta t+V(t, x)+V_{t} \Delta t\right. \\
& +V_{x}\left\{\left[x r(t)+\pi^{\prime}\left(b(t)-r(t) 1_{2}+\lambda(t) \varphi(t)\right)\right.\right. \\
& \left.-\left[\frac{\dot{\alpha}(t)}{\alpha(t)}\left(\pi_{2}\right)^{-}\right]-c\right] \Delta t+\pi^{\prime} \sigma(t) \Delta W(t)+\pi^{\prime} \varphi(t)
\end{aligned}
$$




$$
\begin{aligned}
& \cdot \Delta M(t)\}+\frac{1}{2} V_{t t}(\Delta t)^{2}+\frac{1}{2} V_{x x}\{[x r(t) \\
& +\pi^{\prime}\left(b(t)-r(t) 1_{2}+\lambda(t) \varphi(t)\right)-\left[\frac{\dot{\alpha}(t)}{\alpha(t)}\left(\pi_{2}\right)^{-}\right] \\
& \left.-c] \Delta t+\pi^{\prime} \sigma(t) \Delta W(t)+\pi^{\prime} \varphi(t) \Delta M(t)\right\}^{2} \\
& +V_{t x} \Delta t\left\{\left[x r(t)+\pi^{\prime}\left(b(t)-r(t) 1_{2}+\lambda(t) \varphi(t)\right)\right.\right. \\
& \left.-\left[\frac{\dot{\alpha}(t)}{\alpha(t)}\left(\pi_{2}\right)^{-}\right]-c\right] \Delta t+\pi^{\prime} \sigma(t) \Delta W(t)+\pi^{\prime} \varphi(t) \\
& \cdot \Delta M(t)\}\} .
\end{aligned}
$$

By Karatzas and Shreve [22], it can be obtained that $\mathrm{d} W(t) \cdot \mathrm{d} W(t)=\mathrm{d} t, \mathrm{~d} W(t) \cdot \mathrm{d} t=0, \mathrm{~d} M(t) \cdot \mathrm{d} M(t)=$ $\lambda(t) \mathrm{d} t, \mathrm{~d} M(t) \cdot \mathrm{d} t=0$.

After substituting them into the above equation, we subtract from both sides of the equation $V(t, x)$ divided by $\Delta t$. Finally, by letting $\Delta t \rightarrow 0$, we obtain

$$
\begin{aligned}
V_{t} & +V_{x} x r(t)+\max _{c}\left\{U_{1}(t, c)-c V_{x}\right\} \\
& +\max _{\pi}\left\{V _ { x } \left[\pi^{\prime}\left(b(t)-r(t) 1_{2}+\lambda(t) \varphi(t)\right)\right.\right. \\
& \left.-\left(\frac{\dot{\alpha}(t)}{\alpha(t)}\left(\pi_{2}\right)^{-}\right)\right]+\frac{1}{2} V_{x x}\left[\pi^{\prime} \sigma(t) \sigma(t)^{\prime} \pi\right. \\
& \left.\left.+\lambda(t) \pi^{\prime} \varphi(t) \varphi(t)^{\prime} \pi\right]\right\}=0 .
\end{aligned}
$$

Set $B(t)=b(t)-r(t) 1_{2}+\lambda(t) \varphi(t), D(t)=\sigma(t) \sigma(t)^{\prime}+$ $\lambda(t) \varphi(t) \varphi(t)^{\prime}$. It is assumed that $D(t)$ is positive definite. Substituting them into (16), it is obtained that

$$
\begin{aligned}
V_{t} & +V_{x} x r(t)+\max _{c}\left\{U_{1}(t, c)-c V_{x}\right\} \\
& +\max _{\pi}\left\{V_{x}\left[\pi^{\prime} B(t)-\left(\frac{\dot{\alpha}(t)}{\alpha(t)}\left(\pi_{2}\right)^{-}\right)\right]\right. \\
& \left.+\frac{1}{2} V_{x x} \pi^{\prime} D(t) \pi\right\}=0,
\end{aligned}
$$

with $V(T, x)=U_{2}(T, x)$. Equation (17) is the HJB equation corresponding to (10) and (11).

\section{Optimal Investment-Consumption Strategies}

In this section, the optimal investment-consumption strategies will be derived.
4.1. Optimal Consumption Strategies. From the HJB equation (17), and by applying a first-order optimality condition, we derive that

$$
\frac{\partial\left(U_{1}(t, c)-c V_{x}\right)}{\partial c}=0 .
$$

That is to say, $c^{*}$ satisfies

$$
\frac{\partial U_{1}(t, c)}{\partial c}=V_{x}
$$

4.2. Optimal Investment Strategies. We will also deduce the optimal investment strategies from HJB function (17). Set

$$
\begin{aligned}
f(\pi) & =f\left(\pi_{1}, \pi_{2}\right) \\
& =V_{x}\left[\pi^{\prime} B(t)-\frac{\dot{\alpha}(t)}{\alpha(t)}\left(\pi_{2}\right)^{-}\right]+\frac{1}{2} V_{x x} \pi^{\prime} D(t) \pi, \\
f_{1}(\pi) & =f_{1}\left(\pi_{1}, \pi_{2}\right)=V_{x} \pi^{\prime} B(t)+\frac{1}{2} V_{x x} \pi^{\prime} D(t) \pi, \\
f_{2}(\pi) & =f_{2}\left(\pi_{1}, \pi_{2}\right) \\
& =V_{x}\left[\pi^{\prime} B(t)+\pi_{2} \frac{\dot{\alpha}(t)}{\alpha(t)}\right]+\frac{1}{2} V_{x x} \pi^{\prime} D(t) \pi .
\end{aligned}
$$

By setting $B_{1}(t)=B(t)$ and $B_{2}(t)=B(t)+(0, \dot{\alpha}(t) / \alpha(t))^{\prime}$, the above three equations can be written as follows:

$$
\begin{aligned}
f(\pi)= & f\left(\pi_{1}, \pi_{2}\right) \\
= & V_{x}\left[\pi^{\prime} B_{1}(t)-\frac{\dot{\alpha}(t)}{\alpha(t)}\left(\pi_{2}\right)^{-}\right] \\
& +\frac{1}{2} V_{x x} \pi^{\prime} D(t) \pi, \\
f_{1}(\pi)= & f_{1}\left(\pi_{1}, \pi_{2}\right)=V_{x} \pi^{\prime} B_{1}(t)+\frac{1}{2} V_{x x} \pi^{\prime} D(t) \pi, \\
f_{2}(\pi)= & f_{2}\left(\pi_{1}, \pi_{2}\right)=V_{x} \pi^{\prime} B_{2}(t)+\frac{1}{2} V_{x x} \pi^{\prime} D(t) \pi,
\end{aligned}
$$

thus getting the following equation

$$
f(\pi)=f\left(\pi_{1}, \pi_{2}\right)= \begin{cases}f_{1}\left(\pi_{1}, \pi_{2}\right), & \text { if } \pi_{2}>0, \\ f_{2}\left(\pi_{1}, \pi_{2}\right), & \text { if } \pi_{2} \leq 0 .\end{cases}
$$

From (22), by applying the first-order unconstrained optimality condition, we obtain

$$
\begin{aligned}
\frac{\partial f_{1}(\pi)}{\partial \pi} & =0 \Longrightarrow \\
\hat{\pi} & =-\frac{V_{X}}{V_{X X}} D(t)^{-1} B_{1}(t),
\end{aligned}
$$

and $f_{1}(\tilde{\pi})=-\left(\left(V_{X}\right)^{2} / 2 V_{X X}\right) B_{1}(t)^{\prime} D(t)^{-1} B_{1}(t)$. 
Similarly, from (23) we obtain

$$
\begin{aligned}
\frac{\partial f_{2}(\pi)}{\partial \pi} & =0 \Longrightarrow \\
\breve{\pi} & =-\frac{V_{X}}{V_{X X}} D(t)^{-1} B_{2}(t),
\end{aligned}
$$

and $f_{2}(\breve{\pi})=-\left(\left(V_{X}\right)^{2} / 2 V_{X X}\right) B_{2}(t)^{\prime} D(t)^{-1} B_{2}(t)$.

Next, let us compare $\bar{\pi}$ with $\breve{\pi}$. We have

$$
\tilde{\pi}-\breve{\pi}=\frac{V_{X}}{V_{X X}} D(t)^{-1}\left(0, \frac{\dot{\alpha}(t)}{\alpha(t)}\right)^{\prime} .
$$

By Merton [16], $V(t, x)$ is concave function because the utility functions $U_{1}(\cdot)$ and $U_{2}(\cdot)$ are risk aversion functions. Then, $V_{x} \geq 0, V_{x x}<0$, and $D(t)$ is positive definite. So $\widehat{\pi}_{2}-$ $\breve{\pi}_{2} \leq 0$. We will consider three cases.

(1) $\bar{\pi}_{2}>0$. For any $\pi \leq 0$, we have

$$
\begin{aligned}
f_{2}(\pi) & =V_{x} \pi^{\prime} B_{2}(t)+\frac{1}{2} V_{x x} \pi^{\prime} D(t) \pi \\
& \leq V_{x} \pi^{\prime} B_{1}(t)+\frac{1}{2} V_{x x} \pi^{\prime} D(t) \pi=f_{1}(\pi) \\
& \leq f_{1}(\tilde{\pi}) .
\end{aligned}
$$

Therefore, $\max f(\pi)=\max f_{1}(\pi)=f_{1}(\tilde{\pi})=-\left(\left(V_{X}\right)^{2} /\right.$ $\left.2 V_{X X}\right) B_{1}(t)^{\prime} D(t)^{-1} B_{1}(t)$, and

$$
\pi^{*}=\widehat{\pi}=-\frac{V_{X}}{V_{X X}} D(t)^{-1} B_{1}(t) .
$$

The HJB function (17) can be written as follows:

$$
\begin{aligned}
& V_{t}+V_{x} x r(t)+U_{1}\left(t, c^{*}\right)-c^{*} V_{x} \\
& -\frac{\left(V_{X}\right)^{2}}{2 V_{X X}} B_{1}(t)^{\prime} D(t)^{-1} B_{1}(t)=0, \\
& V(T, x)=U_{2}(T, x) .
\end{aligned}
$$

(2) $\tilde{\pi}_{2} \leq 0 \leq \breve{\pi}_{2}$. The two optimization problems are

$$
\begin{array}{ll}
\max & f_{1}(\pi) \\
& \pi_{2} \geq 0, \quad t \in[0, T] \\
\max & f_{2}(\pi) \\
& \pi_{2} \leq 0, \quad t \in[0, T] .
\end{array}
$$
have

Solving (32), by introducing the Lagrange multiplier $l$, we

$$
L_{1}\left(\pi_{1}, \pi_{2}, l\right)=V_{x} \pi^{\prime} B_{1}(t)+\frac{1}{2} V_{x x} \pi^{\prime} D(t) \pi-l \cdot \pi_{2},
$$

where $l \geq 0$. Then

$$
\frac{\partial L_{1}\left(\pi_{1}, \pi_{2}, l\right)}{\partial \pi_{1}}=V_{x} B_{1}(t)+V_{x x} D(t) \pi=0 .
$$

Considering $\widehat{\pi}_{2} \leq 0 \leq \breve{\pi}_{2}$ and the constraint of (32), it is obtained that the optimal solution of (32) satisfies $\bar{\pi}_{2}=0$. Substituting it into (35), we can get the following equation:

$$
\begin{aligned}
& V_{x}\left(b(t)-r(t)+\lambda(t) \varphi_{1}(t)\right) \\
& \quad+V_{X X}\left(\sigma_{1}^{2}(t)+\lambda(t) \varphi_{1}^{2}(t)\right) \bar{\pi}_{1}=0 .
\end{aligned}
$$

Then $\bar{\pi}_{1}=-\left(V_{X} / V_{X X}\right) \cdot\left(b(t)-r(t)+\lambda(t) \varphi_{1}(t)\right) /\left(\sigma_{1}^{2}(t)+\right.$ $\left.\lambda(t) \varphi_{1}^{2}(t)\right)$.

Following the same line of argument, the solution of (33) is $\bar{\pi}_{2}=0, \bar{\pi}_{1}=-\left(V_{X} / V_{X X}\right) \cdot\left(b(t)-r(t)+\lambda(t) \varphi_{1}(t)\right) /\left(\sigma_{1}^{2}(t)+\right.$ $\left.\lambda(t) \varphi_{1}^{2}(t)\right)$.

As the above discussion mentioned, it is shown that

$$
\pi^{*}=\bar{\pi}=\left[\begin{array}{c}
-\frac{V_{X}}{V_{X X}} \cdot \frac{b_{1}(t)-r(t)+\lambda(t) \varphi_{1}(t)}{\sigma_{1}^{2}(t)+\lambda(t) \varphi_{1}^{2}(t)} \\
0
\end{array}\right] .
$$

Therefore, $\max f(\pi)=f_{1}(\bar{\pi})=-\left(\left(V_{X}\right)^{2} / 2 V_{X X}\right) \cdot((b(t)-$ $\left.\left.r(t)+\lambda(t) \varphi_{1}(t)\right)^{2}\right) /\left(\sigma_{1}^{2}(t)+\lambda(t) \varphi_{1}^{2}(t)\right)$.

Setting $H(t)^{2}=\left(\left(b_{1}(t)-r(t)+\lambda(t) \varphi_{1}(t)\right)^{2}\right) /\left(\sigma_{1}^{2}(t)+\right.$ $\left.\lambda(t) \varphi_{1}^{2}(t)\right)$, the above equation can be written as follows:

$$
\max f(\pi)=f_{1}(\bar{\pi})=-\frac{\left(V_{X}\right)^{2}}{2 V_{X X}} H(t)^{2} .
$$

The HJB function (17) can be written as follows:

$$
V_{t}+V_{x} x r(t)+U_{1}\left(t, c^{*}\right)-c^{*} V_{x}-\frac{\left(V_{X}\right)^{2}}{2 V_{X X}} H(t)^{2}=0,
$$

and $V(T, x)=U_{2}(T, x)$.

(3) $\breve{\pi}_{2}<0$. For any $\pi \leq 0$, we have

$$
\begin{aligned}
f_{1}(\pi) & =V_{x} \pi^{\prime} B_{1}(t)+\frac{1}{2} V_{x x} \pi^{\prime} D(t) \pi \\
& \leq V_{x} \pi^{\prime} B_{2}(t)+\frac{1}{2} V_{x x} \pi^{\prime} D(t) \pi=f_{2}(\pi) \\
& \leq f_{2}(\breve{\pi}) .
\end{aligned}
$$

Therefore, $\max f(\pi)=\max f_{2}(\pi)=f_{1}(\tilde{\pi})=-\left(\left(V_{X}\right)^{2} /\right.$ $\left.2 V_{X X}\right) B_{2}(t)^{\prime} D(t)^{-1} B_{2}(t)$, and

$$
\pi^{*}=\breve{\pi}=-\frac{V_{X}}{V_{X X}} D(t)^{-1} B_{2}(t) .
$$

The HJB function (17) can be written as follows:

$$
\begin{aligned}
V_{t}+ & V_{x} x r(t)+U_{1}\left(t, c^{*}\right)-c^{*} V_{x} \\
- & \frac{\left(V_{X}\right)^{2}}{2 V_{X X}} B_{2}(t)^{\prime} D(t)^{-1} B_{2}(t)=0,
\end{aligned}
$$

and $V(T, x)=U_{2}(T, x)$.

Next, we will discuss the conditions which lead to the above classifications. 


$$
\begin{aligned}
& \widehat{\pi}=-\frac{V_{x}}{V_{x x}} D(t)^{-1} B_{1}(t)=-\frac{V_{x}}{V_{x x}}\left(\sigma(t) \sigma(t)^{\prime}+\lambda(t) \varphi(t) \varphi(t)^{\prime}\right)^{-1}\left(b(t)-r(t) 1_{2}+\lambda(t) \varphi(t)\right) \\
& =-\frac{V_{x}}{V_{x x}}\left[\begin{array}{cc}
\sigma_{1}^{2}(t)+\lambda(t) \varphi_{1}^{2}(t) & \sigma_{1}(t) \sigma_{2}(t)+\lambda(t) \varphi_{1}(t) \varphi_{2}(t) \\
\sigma_{1}(t) \sigma_{2}(t)+\lambda(t) \varphi_{1}(t) \varphi_{2}(t) & \sigma_{2}^{2}(t)+\lambda(t) \varphi_{2}^{2}(t)
\end{array}\right]^{-1} \cdot\left[\begin{array}{l}
b_{1}(t)-r(t)+\lambda(t) \varphi_{1}(t) \\
b_{2}(t)-r(t)+\lambda(t) \varphi_{2}(t)
\end{array}\right] \\
& =-\frac{V_{x}}{V_{x x}}\left[\frac{\iota_{1}-\iota_{2}}{\lambda(t)\left(\sigma_{1}(t) \varphi_{2}(t)-\lambda(t) \sigma_{2}(t) \varphi_{1}(t)\right)^{2}}\right] \\
& \breve{\pi}=-\frac{V_{x}}{V_{x x}} D(t)^{-1} B_{2}(t)=-\frac{V_{x}}{V_{x x}}\left(\sigma(t) \sigma(t)^{\prime}+\lambda(t) \varphi(t) \varphi(t)^{\prime}\right)^{-1}\left(b(t)-r(t) 1_{2}+\lambda(t) \varphi(t)+\left(0, \frac{\dot{\alpha}(t)}{\alpha(t)}\right)^{\prime}\right) \\
& =-\frac{V_{x}}{V_{x x}}\left[\begin{array}{cc}
\sigma_{1}^{2}(t)+\lambda(t) \varphi_{1}^{2}(t) & \sigma_{1}(t) \sigma_{2}(t)+\lambda(t) \varphi_{1}(t) \varphi_{2}(t) \\
\sigma_{1}(t) \sigma_{2}(t)+\lambda(t) \varphi_{1}(t) \varphi_{2}(t) & \sigma_{2}^{2}(t)+\lambda(t) \varphi_{2}^{2}(t)
\end{array}\right]^{-1} \cdot\left[\begin{array}{c}
b_{1}(t)-r(t)+\lambda(t) \varphi_{1}(t) \\
b_{2}(t)-r(t)+\lambda(t) \varphi_{2}(t)+\frac{\dot{\alpha}(t)}{\alpha(t)}
\end{array}\right] \\
& =-\frac{V_{x}}{V_{x x}}\left[\begin{array}{c}
\frac{\iota_{1}-\left(\iota_{2}+\left(\sigma_{1}(t) \sigma_{2}(t)+\lambda(t) \varphi_{1}(t) \varphi_{2}(t)\right)(\dot{\alpha}(t) / \alpha(t))\right)}{\lambda(t)\left(\sigma_{1}(t) \varphi_{2}(t)-\sigma_{2}(t) \varphi_{1}(t)\right)^{2}} \\
\frac{\left(\gamma_{1}+\left(\sigma_{1}^{2}(t)+\lambda(t) \varphi_{1}^{2}(t)\right)(\dot{\alpha}(t) / \alpha(t))\right)-\gamma_{2}}{\lambda(t)\left(\sigma_{1}(t) \varphi_{2}(t)-\sigma_{2}(t) \varphi_{1}(t)\right)^{2}}
\end{array}\right],
\end{aligned}
$$

where

$$
\begin{aligned}
\iota_{1}= & \left(\sigma_{1}^{2}(t)+\lambda(t) \varphi_{2}^{2}(t)\right)\left(b_{1}(t)-r(t)+\lambda(t) \varphi_{1}(t)\right), \\
\iota_{2}= & \left(\sigma_{1}(t) \sigma_{2}(t)+\lambda(t) \varphi_{1}(t) \varphi_{2}(t)\right) \\
& \cdot\left(b_{2}(t)-r(t)+\lambda(t) \varphi_{2}(t)\right), \\
\lambda_{1}= & \left(\sigma_{1}^{2}(t)+\lambda(t) \varphi_{1}^{2}(t)\right) \\
& \cdot\left(b_{2}(t)-r(t)+\lambda(t) \varphi_{2}(t)\right), \\
\lambda_{2}= & \left(\sigma_{1}(t) \sigma_{2}(t)+\lambda(t) \varphi_{1}(t) \varphi_{2}(t)\right) \\
& \cdot\left(b_{1}(t)-r(t)+\lambda(t) \varphi_{1}(t)\right) .
\end{aligned}
$$

Then the optimal investment strategies of (10) and (11) can be written as follows:

$$
\begin{aligned}
& \pi^{*} \\
& \bar{\pi}, \quad \text { if } \gamma_{1}<\gamma_{2} \leq \gamma_{1}+\left(\sigma_{1}^{2}(t)+\lambda(t) \varphi_{1}^{2}(t)\right) \frac{\dot{\alpha}(t)}{\alpha(t)} \\
& \bar{\pi}, \quad \text { if } \gamma_{1}+\left(\sigma_{1}^{2}(t)+\lambda(t) \varphi_{1}^{2}(t)\right) \frac{\dot{\alpha}(t)}{\alpha(t)} \leq \gamma_{2} .
\end{aligned}
$$

\section{HARA Utility Function}

Generally speaking, the analytical solutions to (30), (39), and (42) can not be obtained easily. However, the analytical solutions to the above HJB equations can be derived when the utility function is a HARA function [16]. Moreover, the optimal investment-consumption strategies can be given. Therefore, we assume that both utility functions of (11) are HARA utility functions; that is,

$$
U_{1}(t, y)=U_{2}(t, y)=e^{-\rho t} \frac{y^{\kappa}}{\kappa},
$$

where $0<\kappa<1, \rho>0$ is a discounted factor.

We can get the optimal consumption strategies from (19):

$$
c^{*}=\left(e^{\rho t} V_{x}\right)^{1 /(\kappa-1)} .
$$

Then

$$
\begin{aligned}
\max _{c}\left\{U_{1}(t, c)-c V_{x}\right\}= & U_{1}\left(t, c^{*}\right)-c^{*} V_{x} \\
= & \frac{1}{\kappa} e^{-\rho t}\left(e^{\rho t} V_{x}\right)^{\kappa /(\kappa-1)} \\
& -\left(e^{\rho t} V_{x}\right)^{1 /(\kappa-1)} V_{x} \\
= & \frac{1-\kappa}{\kappa} e^{\rho t /(\kappa-1)}\left(V_{x}\right)^{\kappa /(\kappa-1)} .
\end{aligned}
$$

Next, we will derive the optimal investment strategies. 
(1) $\tilde{\pi} \geq 0$. The HJB function (30) can be written as follows:

$$
\begin{array}{r}
V_{t}+V_{x} x r(t)+\frac{1-\kappa}{\kappa} e^{\rho t /(\kappa-1)}\left(V_{x}\right)^{\kappa /(\kappa-1)} \\
-\frac{\left(V_{X}\right)^{2}}{2 V_{X X}} B_{1}(t)^{\prime} D(t)^{-1} B_{1}(t)=0
\end{array}
$$

and $V(T, x)=e^{-\rho T}\left(x^{\kappa} / \kappa\right)$.

By Merton [16], letting $V(t, x)=\beta_{1}(t)\left(x^{\kappa} / \kappa\right)$ and substituting it into (49), we can obtain

$$
\begin{aligned}
& \beta_{1}(t) \\
& +\kappa\left[r(t)+\frac{1}{2(1-\kappa)} B_{1}(t)^{\prime} D(t)^{-1} B_{1}(t)\right] \beta_{1}(t) \\
& +(1-\kappa) e^{\rho t /(\kappa-1)}\left(\beta_{1}(t)\right)^{\kappa /(\kappa-1)}=0, \\
& \beta_{1}(T)=e^{-\rho T} .
\end{aligned}
$$

Setting

$$
\begin{aligned}
m_{1}(t) & =\kappa\left[r(t)+\frac{1}{2(1-\kappa)} B_{1}(t)^{\prime} D(t)^{-1} B_{1}(t)\right], \\
n(t) & =(1-\kappa) e^{\rho t /(\kappa-1)}
\end{aligned}
$$

and substituting them into (50), we can obtain

$$
\begin{aligned}
\beta_{1}(t)+m_{1}(t) \beta_{1}(t)+n(t)\left(\beta_{1}(t)\right)^{\kappa /(\kappa-1)} & =0, \\
\beta_{1}(T) & =e^{-\rho T} .
\end{aligned}
$$

Setting $\eta_{1}(t)=\beta_{1}(t) e^{-\int_{t}^{T} m_{1}(s) \mathrm{d} s}$ and substituting it into (52), we can get

$$
\begin{aligned}
\eta_{1}(t)+n(t)\left(\eta_{1}(t)\right)^{\kappa /(\kappa-1)} e^{(1 /(\kappa-1)) \int_{t}^{T} m_{1}(s) \mathrm{d} s} & =0, \\
\eta_{1}(T) & =e^{-\rho T} .
\end{aligned}
$$

By solving it, we can obtain

$$
\begin{aligned}
\eta_{1}(t) & =\left(e^{\rho T /(\kappa-1)}\right. \\
& \left.+\frac{1}{1-\kappa} \int_{t}^{T} n(s) e^{(1 /(\kappa-1)) \int_{s}^{T} m_{1}(v) \mathrm{d} v} \mathrm{~d} s\right)^{1-\kappa} .
\end{aligned}
$$

Then

$$
\begin{aligned}
& \beta_{1}(t)=e^{\int_{t}^{T} m_{1}(s) \mathrm{d} s}\left(e^{\rho T /(\kappa-1)}\right. \\
& \left.+\frac{1}{1-\kappa} \int_{t}^{T} n(s) e^{(1 /(\kappa-1)) \int_{s}^{T} m_{1}(v) \mathrm{d} v} \mathrm{~d} s\right)^{1-\kappa} .
\end{aligned}
$$

Substituting $V(t, x)=\beta_{1}(t)\left(x^{\kappa} / \kappa\right)$ into (29) and (47), we can get the optimal investment-consumption strategies as follows:

$$
\begin{aligned}
\pi^{*} & =\hat{\pi}=-\frac{D(t)^{-1} B_{1}(t)}{\kappa-1} x, \\
c^{*} & =e^{\rho t /(\kappa-1)}\left(\beta_{1}(t)\right)^{1 /(\kappa-1)} x .
\end{aligned}
$$

(2) $\tilde{\pi} \leq 0 \leq \breve{\pi}$. The HJB function (39) can be written as follows:

$$
\begin{gathered}
V_{t}+V_{x} x r(t)+\frac{1-\kappa}{\kappa} e^{\rho t /(\kappa-1)}\left(V_{x}\right)^{\kappa /(\kappa-1)} \\
-\frac{\left(V_{X}\right)^{2}}{2 V_{X X}} H(t)^{2}=0,
\end{gathered}
$$

and $V(T, x)=e^{-\rho T}\left(x^{\kappa} / \kappa\right)$.

Similar to (1), we can obtain by letting $V(t, x)=\beta_{1}(t)\left(x^{\kappa} /\right.$ $\kappa)$ and substituting it into (57)

$$
\begin{aligned}
& \beta_{1}(t)+\kappa\left[r(t)+\frac{1}{2(1-\kappa)} H(t)^{2}\right] \beta_{1}(t) \\
& +(1-\kappa) e^{\rho t /(\kappa-1)}\left(\beta_{1}(t)\right)^{\kappa /(\kappa-1)}=0, \\
& \beta_{1}(T)=e^{\rho T} .
\end{aligned}
$$

Setting

$$
m_{2}(t)=\kappa\left[r(t)+\frac{1}{2(1-\kappa)} H(t)^{2}\right]
$$

and substituting it into (58), we can obtain

$$
\begin{aligned}
\beta_{2}(t)+m_{2}(t) \beta_{2}(t)+n(t)\left(\beta_{2}(t)\right)^{\kappa /(\kappa-1)} & =0, \\
\beta_{2}(T) & =e^{-\rho T} .
\end{aligned}
$$

Setting $\eta_{2}(t)=\beta_{2}(t) e^{-\int_{t}^{T} m_{2}(s) \mathrm{d} s}$ and substituting it into (52), we can get

$$
\begin{aligned}
\eta_{2}(t)+n(t)\left(\eta_{2}(t)\right)^{\kappa /(\kappa-1)} e^{(1 /(\kappa-1)) \int_{t}^{T} m_{2}(s) \mathrm{d} s} & =0, \\
\eta_{2}(T) & =e^{-\rho T} .
\end{aligned}
$$

By solving it, we can obtain

$$
\begin{aligned}
\eta_{2}(t) & =\left(e^{\rho T /(\kappa-1)}\right. \\
+ & \left.\frac{1}{1-\kappa} \int_{t}^{T} n(s) e^{(1 /(\kappa-1)) \int_{s}^{T} m_{2}(v) \mathrm{d} v} \mathrm{~d} s\right)^{1-\kappa}
\end{aligned}
$$

Then

$$
\begin{aligned}
& \beta_{2}(t)=e^{\int_{t}^{T} m_{2}(s) \mathrm{d} s}\left(e^{\rho T /(\kappa-1)}\right. \\
& \left.+\frac{1}{1-\kappa} \int_{t}^{T} n(s) e^{(1 /(\kappa-1)) \int_{s}^{T} m_{2}(v) \mathrm{d} v} \mathrm{~d} s\right)^{1-\kappa}
\end{aligned}
$$


By substituting $V(t, x)=\beta_{2}(t)\left(x^{\kappa} / \kappa\right)$ into (37) and (47), we can get the optimal investment-consumption strategies as follows:

$$
\begin{aligned}
& \pi^{*}=\widehat{\pi}=\left[\begin{array}{c}
-\frac{b_{1}(t)-r(t)+\lambda(t) \varphi_{1}(t)}{\sigma_{1}^{2}(t)+\lambda(t) \varphi_{1}^{2}(t)} \cdot \frac{x}{\kappa-1} \\
0
\end{array}\right], \\
& c^{*}=e^{\rho t /(\kappa-1)}\left(\beta_{2}(t)\right)^{1 /(\kappa-1)} x .
\end{aligned}
$$

(3) $\breve{\pi} \leq 0$. The HJB function (42) can be written as follows:

$$
\begin{array}{r}
V_{t}+V_{x} x r(t)+\frac{1-\kappa}{\kappa} e^{\rho t /(\kappa-1)}\left(V_{x}\right)^{\kappa /(\kappa-1)} \\
-\frac{\left(V_{X}\right)^{2}}{2 V_{X X}} B_{2}(t)^{\prime} D(t)^{-1} B_{2}(t)=0
\end{array}
$$

and $V(T, x)=e^{-\rho T}\left(x^{\kappa} / \kappa\right)$.

Similar to (1), we can obtain by letting $V(t, x)=\beta_{3}(t)\left(x^{\kappa} /\right.$ $\kappa)$ and substituting it into (64)

$$
\begin{aligned}
& \beta_{3}(t) \\
& +\kappa\left[r(t)+\frac{1}{2(1-\kappa)} B_{2}(t)^{\prime} D(t)^{-1} B_{2}(t)\right] \beta_{3}(t) \\
& +(1-\kappa) e^{\rho t /(\kappa-1)}\left(\beta_{3}(t)\right)^{\kappa /(\kappa-1)}=0, \\
& \beta_{3}(T)=e^{-\rho T} .
\end{aligned}
$$

Setting

$$
m_{3}(t)=\kappa\left[r(t)+\frac{1}{2(1-\kappa)} B_{2}(t)^{\prime} D(t)^{-1} B_{2}(t)\right]
$$

$$
\begin{aligned}
& \pi^{*}= \begin{cases}-\frac{D(t)^{-1} B_{1}(t)}{\kappa-1} x, & \text { if } \gamma_{1} \geq \gamma_{2} ; \\
{\left[\begin{array}{ll}
-\frac{b_{1}(t)-r(t)+\lambda(t) \varphi_{1}(t)}{\sigma_{1}^{2}(t)+\lambda(t) \varphi_{1}^{2}(t)} \cdot \frac{x}{\kappa-1} \\
0
\end{array}\right],} & \text { if } \gamma_{1}<\gamma_{2} \leq \gamma_{1}+\left(\sigma_{1}^{2}(t)+\lambda(t) \varphi_{1}^{2}(t)\right) \frac{\dot{\alpha}(t)}{\alpha(t)} ; \\
-\frac{D(t)^{-1} B_{2}(t)}{\kappa-1} x, & \text { if } \gamma_{1}+\left(\sigma_{1}^{2}(t)+\lambda(t) \varphi_{1}^{2}(t)\right) \frac{\dot{\alpha}(t)}{\alpha(t)} \leq \gamma_{2},\end{cases} \\
& c^{*}= \begin{cases}e^{\rho t /(\kappa-1)}\left(\beta_{1}(t)\right)^{1 /(\kappa-1)} x, & \text { if } \gamma_{1} \geq \gamma_{2} ; \\
e^{\rho t /(\kappa-1)}\left(\beta_{2}(t)\right)^{1 /(\kappa-1)} x, & \text { if } \gamma_{1}<\gamma_{2} \leq \gamma_{1}+\left(\sigma_{1}^{2}(t)+\lambda(t) \varphi_{1}^{2}(t)\right) \frac{\dot{\alpha}(t)}{\alpha(t)} \\
e^{\rho t /(\kappa-1)}\left(\beta_{3}(t)\right)^{1 /(\kappa-1)} x, & \text { if } \gamma_{1}+\left(\sigma_{1}^{2}(t)+\lambda(t) \varphi_{1}^{2}(t)\right) \frac{\dot{\alpha}(t)}{\alpha(t)} \leq \gamma_{2} .\end{cases}
\end{aligned}
$$


From (73) and (74), it can be seen that the results are closed-form solutions. We can avoid solving the partial differential equation difficulty and may obtain the optimal investment-consumption policies corresponding capital $x$ directly.

\section{A Numerical Example and Discussion}

In this section, we will introduce a numerical example to illustrate model (11) with HARA utility function (46). This example is only to show how to obtain the optimal investment-consumption strategies. Some parameters have been simplified to some extent. We can change the assumptions of the parameters to obtain the results which would deliver the different $\gamma_{1}$ and $\gamma_{2}$.

(1) It is assumed that the initial capital is $x_{0}=1.19 \times$ $10^{5} \mathrm{RMB}$. We only calculate the capital range $\left[x_{0}, 2 x_{0}\right]$ for illustrating the model. The rest of the parameters are as follows:

$$
\begin{aligned}
\rho & =0.02, \\
\kappa & =0.5, \\
r & =0.025, \\
\lambda & =0.6, \\
\omega & =0.07, \\
\theta & =0.39, \\
\zeta & =0.1 .
\end{aligned}
$$

For simplicity, we set $T=1$. In a given time $t=0.5$, the futures price $F=53 \mathrm{RMB}$ and its corresponding Europe call option price is $Y=4.77 \mathrm{RMB}$. We also suppose that $Y_{t}=0.13, Y_{F}=0.23, Y_{F F}=-0.0123, \alpha=$ $0.85, \dot{\alpha}=1$ on this moment. In the above parameters, $\gamma_{1}<\gamma_{2} \leq \gamma_{1}+\left(\sigma_{1}^{2}(t)+\lambda(t) \varphi_{1}^{2}(t)\right)(\dot{\alpha}(t) / \alpha(t))$, the optimal investment-consumption strategies are illustrated as Figure $1(1.21 E+5$ means 121,000 , the same below). From Figure 1, we can see that we do not invest in options anymore. The investor can only allocate his/her little capital in the futures. A large number capital is invested in the risk-free bond.

(2) We change parameters $\dot{\alpha}=0.005$ and $\zeta=0.9$ from comparing with (1). In the case $\gamma_{1}+\left(\sigma_{1}^{2}(t)+\right.$ $\left.\lambda(t) \varphi_{1}^{2}(t)\right)(\dot{\alpha}(t) / \alpha(t)) \leq \gamma_{2}$, the optimal investmentconsumption strategies will change as shown in Figure 2. Figure 2 makes us purchase futures and sell the options.

(3) If $\zeta=0.1, Y_{F F}=0.005$, and $\omega=0.07$, then $\gamma_{1} \geq \gamma_{2}$ comparing with (1). The optimal investmentconsumption strategies are illustrated in Figure 3, which shows how the investors could sell futures and purchase the options.

Although this example is simple, it well explains the sense of the model. From the computed results, the optimal

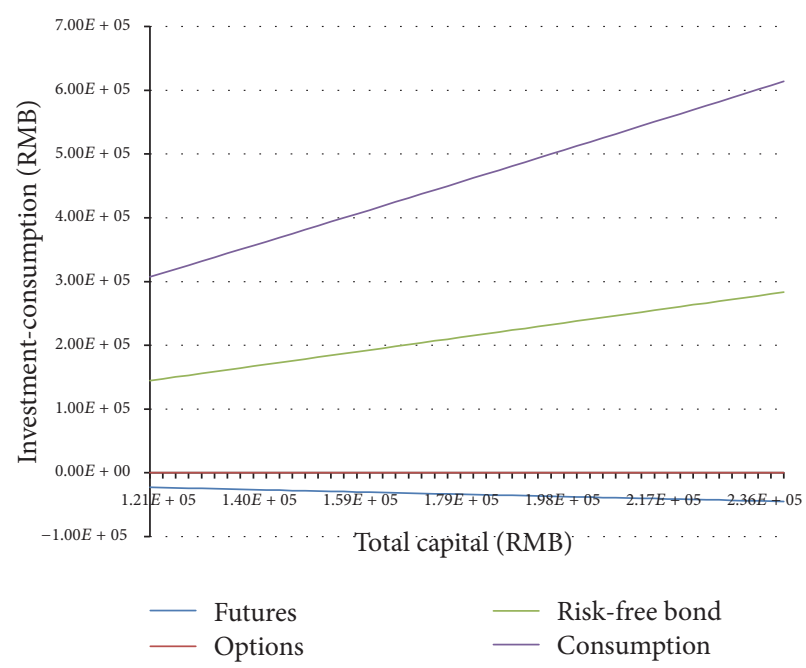

Figure 1: Optimal strategies under $\gamma_{1}<\gamma_{2} \leq \gamma_{1}+\left(\sigma_{1}^{2}(t)+\right.$ $\left.\lambda(t) \varphi_{1}^{2}(t)\right)(\dot{\alpha}(t) / \alpha(t))$.

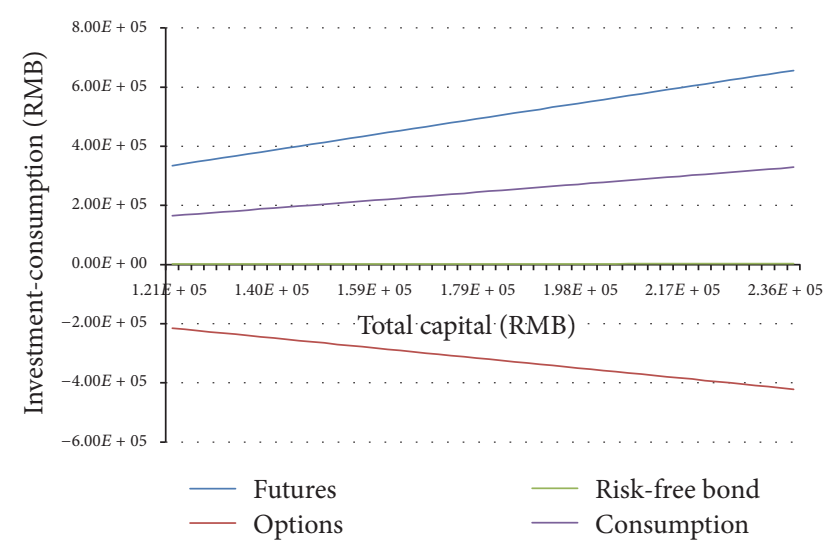

Figure 2: Optimal strategies under $\gamma_{1}+\left(\sigma_{1}^{2}(t)+\lambda(t) \varphi_{1}^{2}(t)\right)(\dot{\alpha}(t) /$ $\alpha(t)) \leq \gamma_{2}$.

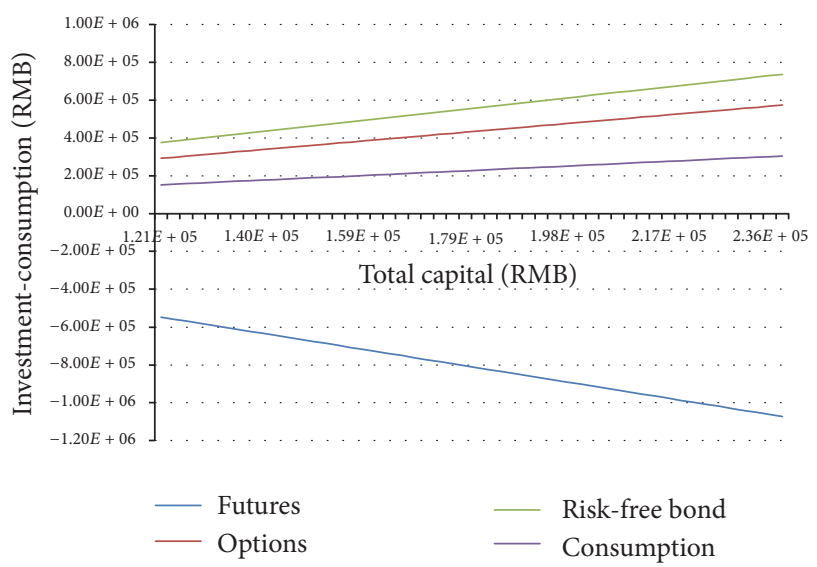

FIGURE 3: Optimal strategies under $\gamma_{1} \geq \gamma_{2}$. 
investment-consumption strategy is a closed-form solution, thus avoiding solving partial differential equations numerically. We remark that the numerical solution of partial differential equations can be a challenging task. The closedform analytical solution can greatly improve the calculation speed and reduce the computational complexity. However, you need to make some assumptions of stochastic differential equation parameters if you may also find more details. The focus of this paper is the theoretical results rather than numerical calculations. Therefore, the above example is a simulation which further describes the theoretical results.

Comparing with the classical Merton model, the Poisson process is added to the portfolio with Brown motion process. The results are very similar to the traditional Merton solution outwardly. However, the jump process has greatly increased the complexity of the solution process. For utility functions similar to those considered in this paper, relevance of capital between optimal strategies can be obtained after solving the optimal consumption-investment strategies from (73), (74), or the above examples. The investors can differently allocate their capital by adjusting the parameters of utility function.

\section{Conclusions}

We study the optimal investment-consumption portfolio selection of options which have a different price between sale and purchase based on a risk aversion utility function in financial markets. These pricing processes follow a Weiner process and a Poisson process. Then the corresponding HJB equation of the problem is derived and its solutions are obtained in different conditions, respectively. Finally, a special case, when the utility function is HARA, is illustrated. In addition, throughout the paper it is assumed that $\alpha(t)$ is differentiable with respect to time $t$ and monotone nondecreasing. Future work will be devoted to the collection of a large number of datasets in the real market for calibrating the various parameters of the model.

\section{Conflicts of Interest}

The author declares that there are no conflicts of interest regarding the publication of this article.

\section{References}

[1] F. Black and M. Scholes, "The pricing of options corporate liabilities," Journal of Political Economy, vol. 81, pp. 637-659, 1973.

[2] R. C. Merton, "Option pricing when underlying stock returns are discontinuous," Journal of Financial Economics, vol. 3, no. 1-2, pp. 125-144, 1976.

[3] M. Jeanblanc-Picque and M. Pontier, "Optimal portfolio for a small investor in a market model with discontinuous prices," Applied Mathematics and Optimization, vol. 22, no. 3, pp. 287$310,1990$.

[4] J. Z. Lin and Z. X. Ye, "The valuation of European contingent claims of several stocks whose prices are governed by Brownian motions and Poisson processes," Chinese Journal of Applied Probability, vol. 18, pp. 167-172, 2002.
[5] E. N. Barron and R. Jensen, "A stochastic control approach to the pricing of options," Mathematics of Operations Research, vol. 15, no. 1, pp. 49-79, 1990.

[6] J. C. Cox, S. A. Ross, and M. Rubinstein, "Option pricing: a simplified approach," Journal of Financial Economics, vol. 7, no. 3, pp. 229-263, 1979.

[7] H. Markowitz, "Portfolio selection," Journal of Finance, vol. 7, pp. 77-91, 1952.

[8] R. C. Merton, "Lifetime portfolio selection under uncertainty: the continuous-time model," The Review of Economics and Statistics, vol. 51, pp. 247-257, 1969.

[9] R. C. Merton, "Optimum consumption and portfolio rules in a continuous-time model," Journal of Economic Theory, vol. 3, no. 4, pp. 373-413, 1971.

[10] X. Li, X. Y. Zhou, and A. E. Lim, "Dynamic mean-variance portfolio selection with no-shorting constraints," SIAM Journal on Control and Optimization, vol. 40, no. 5, pp. 1540-1555, 2002.

[11] A. Cadenillas, "Consumption-investment problems with transaction costs: survey and open problems," Mathematical Methods of Operations Research, vol. 51, no. 1, pp. 43-68, 2000.

[12] X. Y. Zhou and D. Li, "Continuous-time mean-variance portfolio selection: a stochastic LQ framework," Applied Mathematics and Optimization, vol. 42, no. 1, pp. 19-33, 2000.

[13] D. Cuoco and H. Liu, "A martingale characterization of consumption choices and hedging costs with margin requirements," Mathematical Finance. An International Journal of Mathematics, Statistics and Financial Economics, vol. 10, no. 3, pp. 355-385, 2000.

[14] A. E. Lim and X. Y. Zhou, "Mean-variance portfolio selection with random parameters in a complete market," Mathematics of Operations Research, vol. 27, no. 1, pp. 101-120, 2002.

[15] I. Karatzas, J. P. Lehoczky, S. E. Shreve, and G.-L. Xu, "Martingale and duality methods for utility maximization in an incomplete market," SIAM Journal on Control and Optimization, vol. 29, no. 3, pp. 702-730, 1991.

[16] R. C. Merton, Continuous-Time Finance, Basil Blackwell, Cambridge, MA, USA, 1990.

[17] R. A. Jarrow and A. Rudd, Option Pricing, vol. 3, Homewood, 1983.

[18] J. C. Cox and S. A. Ross, "The valuation of options for alternative stochastic processes," Journal of Financial Economics, vol. 3, no. 1-2, pp. 145-166, 1985.

[19] W. Yan and Y. Chang, "Modelling on optimal portfolio with exchange rate based on discontinuous stochastic process," International Journal of Control, vol. 89, no. 12, pp. 2543-2548, 2016.

[20] G. L. Gong, Introduction to Stochastic Differential Equations, Peking University Press, Peking, 1987.

[21] A. P. Sage and C. C. White, Optimum systems control, vol. 3, Prentice-Hall, Englewood Cliffs, N.J., USA, 1977.

[22] I. Karatzas and S. E. Shreve, Brownian Motion and Stochastic Calculus, Springer, New York, NY, USA, 2nd edition, 1988. 


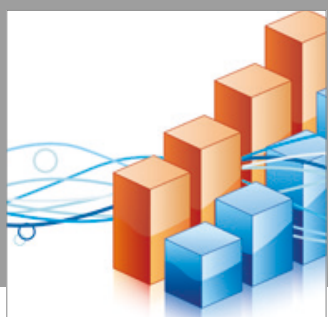

Advances in

Operations Research

vatersals

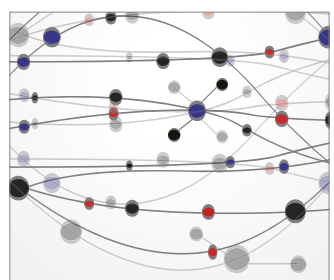

\section{The Scientific} World Journal
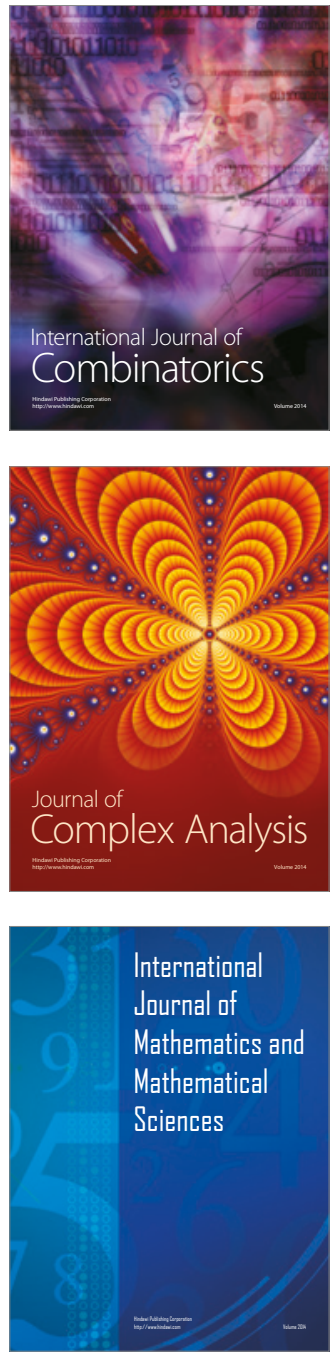
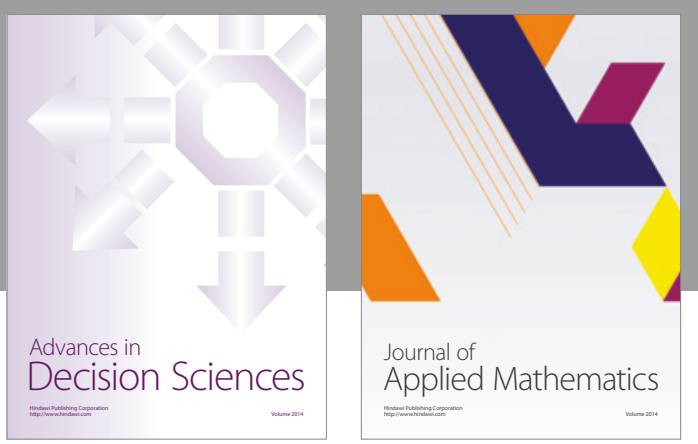

Algebra

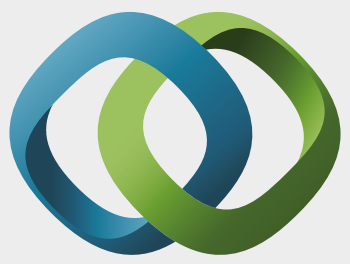

\section{Hindawi}

Submit your manuscripts at

https://www.hindawi.com
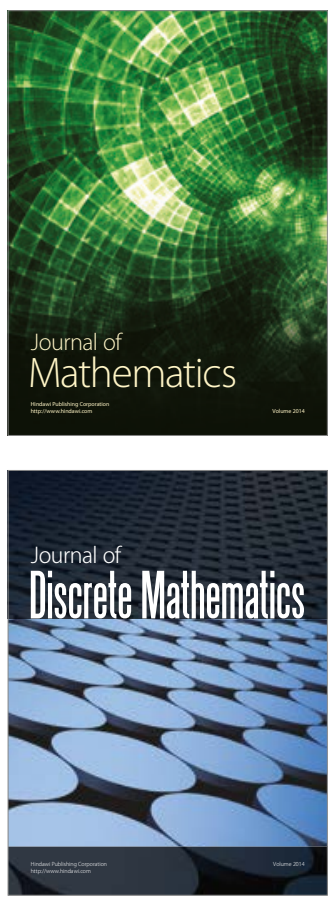

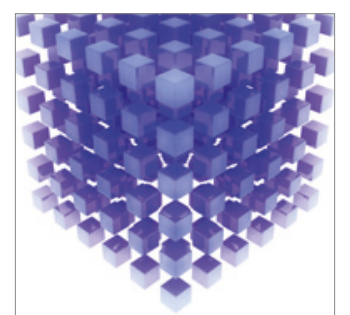

Mathematical Problems in Engineering
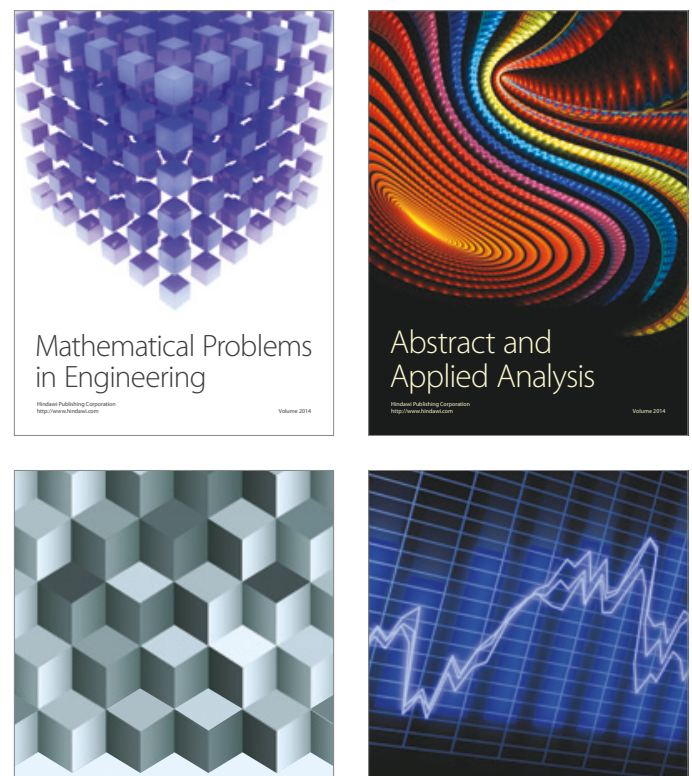

Journal of

Function Spaces

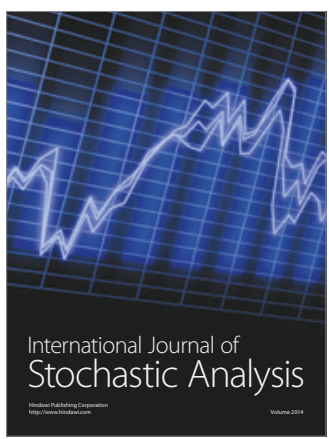

Probability and Statistics
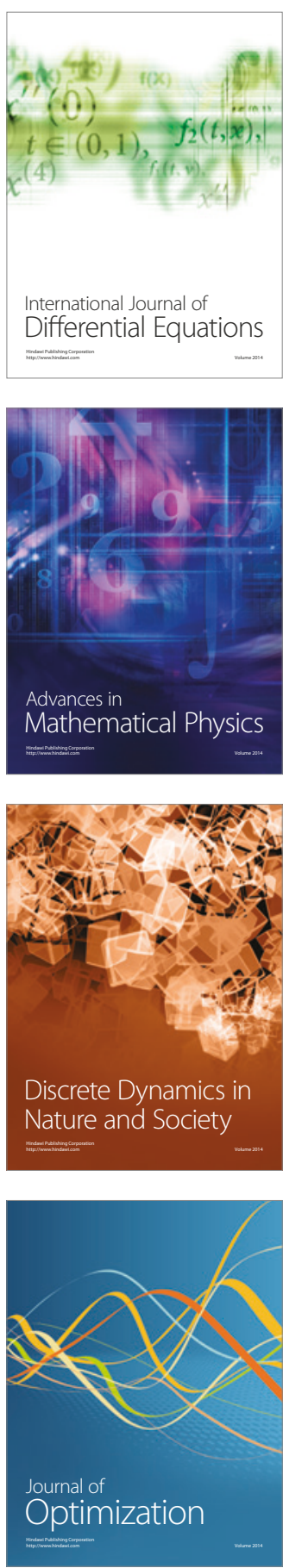